$\underline{\mathbf{P}-120}$

\title{
The Double-Peak Phenomenon and Bioavailability Studies of a Quassinoid-Rich Standardized Extract of Eurycoma Longifolia Solid Dispersion Formulation
}

\author{
Hai-Qiu Ma, Nurzalina Abdul Karim Khan and Kit-Lam Chan* \\ School of Pharmaceutical Sciences, Universiti Sains Malaysia, 11800 Minden, Penang, Malaysia; \\ E-mails:kitlamc@gmail.com; klchan@usm.my
}

The quassinoid-rich standardized extract of Eurycoma longifoia extract (TAF2) was found to possess various biological activities. However, the poor oral bioavailability of the bioactive quassinoids, $13 \alpha(21)$-epoxyeurycomanone (EP) and eurycomanone (EN) in TAF2 necessitated a new formulation design to be developed. A lipid-based solid dispersion was formulated and the bioavailabilities of EP and EN were significant improved compared with the conventional powder-filled capsules. A double peak phenomenon was identified in the plasma concentration-time curve. The gastric emptying and variability of GIT absorption, the probable causes of the double peak phenomenon, were studied by administering TAF2 solid dispersion to Sprague Dawley rats. As marker compounds, theophylline and sulfasalazine were used to determine the gastric emptying and variability of GIT absorption. The percentage absorbed of the markers was calculated using Wagner-Nelson method. The results showed that $50 \%$ of theophylline was absorbed at $3 \mathrm{~h}$, indicating the transit time of TAF 2 solid dispersion from the stomach to the small intestine. Whereas, the $50 \%$ of sulfapyridine, the metabolite of sulfasalazine, was absorbed at 8 $\mathrm{h}$, reflected time of TAF2 solid dispersion to reach the colon. The concentration of TAF2 in colon was $74.36 \%$ for EP and $80.27 \%$ for EN, respectively. The results indicated that the delayed gastric emptying and colon absorption were the causes of double peak phenomenon. Thus, the delayed onset of in vivo activity of the quassinoids in E. longifolia was probably due to the delay of gastric emptying and colon absorption.

Keywords: Eurycoma longifoia, solid dispersion, double peak phenomenon. 